\title{
Minimal exact balancedness
}

Citation for published version (APA):

Lohmann, E., Borm, P., \& Herings, P. J. J. (2012). Minimal exact balancedness. Mathematical Social

Sciences, 64(2), 127-135. https://doi.org/10.1016/j.mathsocsci.2012.01.002

Document status and date:

Published: 01/09/2012

DOI:

10.1016/j.mathsocsci.2012.01.002

Document Version:

Publisher's PDF, also known as Version of record

Document license:
Taverne

\section{Please check the document version of this publication:}

- A submitted manuscript is the version of the article upon submission and before peer-review. There can be important differences between the submitted version and the official published version of record.

People interested in the research are advised to contact the author for the final version of the publication, or visit the DOI to the publisher's website.

- The final author version and the galley proof are versions of the publication after peer review.

- The final published version features the final layout of the paper including the volume, issue and page numbers.

Link to publication

\footnotetext{
General rights rights.

- You may freely distribute the URL identifying the publication in the public portal. please follow below link for the End User Agreement:

www.umlib.nl/taverne-license

Take down policy

If you believe that this document breaches copyright please contact us at:

repository@maastrichtuniversity.nl

providing details and we will investigate your claim.
}

Copyright and moral rights for the publications made accessible in the public portal are retained by the authors and/or other copyright owners and it is a condition of accessing publications that users recognise and abide by the legal requirements associated with these

- Users may download and print one copy of any publication from the public portal for the purpose of private study or research.

- You may not further distribute the material or use it for any profit-making activity or commercial gain

If the publication is distributed under the terms of Article $25 \mathrm{fa}$ of the Dutch Copyright Act, indicated by the "Taverne" license above, 


\title{
Minimal exact balancedness
}

\author{
E. Lohmann ${ }^{\text {a }}$, P. Borm ${ }^{\text {a }}$, P.J.J. Herings ${ }^{\text {b,* }}$ \\ a CentER and Department of Econometrics and OR, Tilburg University, Tilburg, The Netherlands \\ ${ }^{\mathrm{b}}$ Department of Economics, Maastricht University, Maastricht, The Netherlands
}

\section{A R T I C L E I N F O}

Article history:

Available online 15 January 2012

\begin{abstract}
A B S T R A C T
To verify whether a transferable utility game is exact, one has to check a linear inequality for each exact balanced collection of coalitions. This paper studies the structure and properties of the class of exact balanced collections. Comparing the definition of exact balanced collections with the definition of balanced collections, the weight vector of a balanced collection must be positive whereas the weight vector for an exact balanced collection may contain one negative weight. We investigate minimal exact balanced collections, and show that only these collections are needed to obtain exactness. The relation between minimality of an exact balanced collection and uniqueness of the corresponding weight vector is analyzed. We show how the class of minimal exact balanced collections can be partitioned into three basic types each of which can be systematically generated.
\end{abstract}

(C) 2012 Elsevier B.V. All rights reserved.

\section{Introduction}

One of the most important notions in cooperative game theory is the core. Introduced by Gillies (1953), the core consists of all allocations that are both individually and coalitionally stable. Given an allocation in the core of the game, no coalition has an incentive to split off. There exist games for which such an allocation does not exist, resulting in an empty core. Bondareva (1963) and Shapley (1967) showed independently that non-emptiness of the core is equivalent with balancedness.

A collection of coalitions is balanced if one can find positive weights for all coalitions in the collection such that every player is present in coalitions with total weight exactly equal to one. A game is balanced if for all such collections and all such weights, the weighted sum of the values of the coalitions does not exceed the value of the grand coalition. An interpretation is that the players can distribute one unit of working time among all coalitions in such a way that for every coalition, all members are active for an amount of time equal to the coalition's weight, and in doing so the players cannot create more value than by working one unit of time in the grand coalition.

The concept of balanced collections has played a major role in the literature on the nucleolus (Schmeidler, 1969), the prenucleolus (Schmeidler, 1969), and weighted nucleoli (Derks and Haller, 1999). In particular, it is an important part of the Kohlberg

\footnotetext{
* Corresponding author.

E-mail addresses: e.r.m.a.lohmann@uvt.nl (E. Lohmann), p.e.m.borm@uvt.nl (P. Borm), p.herings@maastrichtuniversity.nl (P.J.J. Herings).
}

condition (Kohlberg, 1971), which is used to check if a given imputation is the nucleolus of a given game. Furthermore, balanced collections are strong tools in proofs on properties and characterizations as is seen in e.g., Derks and Haller (1999).

To verify that the core of a game is non-empty, not all balanced collections are needed. A balanced collection of coalitions is minimal, if there does not exist a proper subset that is also balanced. As it turns out, only minimal balanced collections have to be considered to ensure non-emptiness of the core. This greatly reduces the number of constraints to be checked for nonemptiness of the core. Furthermore, the class of minimal balanced collections is sharp, in the sense that there exists no subclass of the class of minimal balanced collections that ensures balancedness of the game.

A game is exact (Schmeidler, 1972) if for every coalition, there exists a core element that allocates precisely the value of the coalition to its members. Therefore in such a core element, the coalition gets exactly its stand alone value. Many important applications of cooperative game theory have led to the study of exact games. Classes of games such as e.g., convex games (Shapley, 1971), risk allocation games with no aggregate uncertainty (Csóka et al., 2009), convex multi-choice games (Branzei et al., 2009) and multi-issue allocation games (Calleja et al., 2005) are exact. Exactness turns out to be equivalent with exact balancedness as introduced in Csóka et al. (2011). Exact balancedness is similar to the notion of balancedness, when we allow one of the weights to be negative.

Regarding exact balancedness, many exact balanced collections are redundant when verifying the exactness of a game. We show that only minimal exact balanced collections are essential to obtain exactness. However, it is not possible to use the same approach as 
with minimal balanced collections. This is due to the fact that while the set of balanced weight vectors is a convex set in which the extreme points are the weight vectors corresponding with minimal balanced collections, the set of exact balanced weight vectors is not a convex set.

We show that the class of minimal exact balanced collections can be partitioned into three types. The first type consists of all minimal balanced sets. The second type, the class of minimal subbalanced collections, is formed by all minimal balanced collections for every proper subgame, to which two coalitions are added: the grand coalition of the subgame, and the grand coalition of the original game. The last type, the class of minimal negative balanced collections, consists of all other minimal exact balanced collections for which every weight vector has one negative weight.

One of the main results concerns the special structure of the class of minimal negative balanced collections. We show that every minimal negative balanced collection can be obtained from a minimal balanced collection by replacing one coalition, with a weight strictly smaller than one, by its complement. Moreover, for every minimal negative balanced collection there exists exactly one such combination of a minimal balanced collection and a coalition with a weight strictly smaller than one.

The class of minimal exact balanced collections ensures exactness of the game, but the class can be reduced even further. We show that only the class of minimal subbalanced collections and the class of minimal negative balanced collections are needed to guarantee exactness. So, the class of minimal balanced collections is redundant.

With respect to the uniqueness of the weights, it is well known that the class of minimal balanced collections coincides with the set of balanced collections for which the set of balanced weight vectors consists of one point. A similar result can be obtained for minimal exact balanced collections. If the exact balanced weight vector is unique for a certain exact balanced collection, then this collection is minimal exact balanced. The other way around is not true in a strict sense. For two types, minimal balanced and minimal negative balanced collections, the corresponding weight vector is unique. For every minimal subbalanced collection however, there exists more than one exact balanced weight vector but all weight vectors are related to each other by a linear transformation, and induce the same constraint on the game.

In the process, we also see how we can systematically and efficiently generate all minimal exact balanced collections, by adapting the inductive approach to construct all minimal balanced collections by Peleg (1965).

Just as balanced collections are not only used to verify the nonemptiness of the core, but also in characterizing the pre-nucleolus useful in several results on (variations of) the nucleolus, these insights in the theoretical structure of exact balanced collections provide a wider range of techniques to obtain further results on these solution concepts.

The paper is organized as follows: the subsequent section introduces some notions regarding cooperative game theory, and repeats the main results regarding balanced collections. Section 3 contains the definitions of several notions regarding exact balancedness, and includes the results on the uniqueness of the weights. Section 4 shows that the class of minimal exact balanced collections can be partitioned into three easily identifiable types. Section 5 states that minimal exact balanced collections are sufficient to ensure exactness of the game. Section 6 describes the construction of minimal exact balanced collections.

\section{Balancedness}

First, we introduce some basic notions regarding cooperative game theory and balancedness. Given a finite player set $N$, a transferable utility game $v \in \mathrm{TU}^{N}$ is defined by a function $v$ on the set $2^{N}$ of all subsets of $N$ assigning to each coalition $S \in 2^{N}$ a value $v(S)$ such that $v(\emptyset)=0$. Define $\mathcal{N}=2^{N} \backslash\{\emptyset\}$, and for all $S \in \mathcal{N}$ let $e^{S} \in \mathbb{R}^{N}$ be such that $e_{i}^{S}=1$ if $i \in S$ and $e_{i}^{S}=0$ otherwise. For a game $v \in \mathrm{TU}^{N}$, the core $C(v)$ is defined as the set of efficient pay-off vectors, for which no coalition has an incentive to split off:

$C(v)=\left\{x \in \mathbb{R}^{N} \mid \sum_{i \in N} x_{i}=v(N), \sum_{i \in S} x_{i} \geq v(S)\right.$ for all $\left.S \in \mathcal{N}\right\}$.

To check for non-emptiness of the core, one can use the notion of balancedness.

Definition 2.1. Let $\mathscr{B} \subseteq \mathcal{N}, \mathscr{B} \neq\{N\}$. A weight vector $\beta \in \mathbb{R}^{\mathcal{N}}$ is called balanced on $\mathscr{B}$ if $\beta_{S}>0$ for all $S \in \mathscr{B}, \beta_{S}=0$ for all $S \notin \mathscr{B}$ and $\sum_{S \in \mathcal{B}} \beta_{S} e^{S}=e^{N}$. We denote the set of all balanced weight vectors on $\mathscr{B}$ by $\Lambda^{+}(\mathscr{B})$. The collection $\mathscr{B}$ is called balanced if $\Lambda^{+}(\mathscr{B}) \neq \emptyset$. Denote $\mathbb{B}^{N}$ for the set of all balanced collections on player set $N$, and $\Lambda^{+}=\cup_{\mathscr{B} \in \mathbb{B}^{N}} \Lambda^{+}(\mathscr{B})$.

In the remainder, we will typically use $\mathcal{B}$ and $\mathcal{C}$ to denote balanced collections, and use $\beta$ and $\gamma$ to denote their respective weight vectors.

Example 2.2. Let $N=\{1,2\}$. The collections $\{\{1\}\}$ and $\{\{2\}\}$ are not balanced, since one of the players is not present in the collection. By definition $\{\{1,2\}\}$ is not balanced. The collection $\{\{1\},\{1,2\}\}$ is not balanced. This follows as a balanced weight vector $\beta$ cannot satisfy the equations $\beta_{\{1,2\}}=1$ and $\beta_{\{1\}}+\beta_{\{1,2\}}=1$ simultaneously, since $\beta_{\{1\}}>0$. A similar reasoning holds for the collection $\{\{2\},\{1,2\}\}$. The two remaining collections are $\mathscr{B}=$ $\{\{1\},\{2\}\}$ and $\mathcal{C}=\{\{1\},\{2\},\{1,2\}\}$, which are both balanced. Take $\beta \in \Lambda^{+}$such that $\beta_{\{1\}}=\beta_{\{2\}}=1$ and $\beta_{S}=0$ for $S \in \mathcal{N} \backslash\{\{1\},\{2\}\}$, and take $\gamma \in \Lambda^{+}$such that $\gamma_{\{1,2\}}=1$ and $\gamma_{S}=0$ for $S \in \mathcal{N} \backslash\{\{1,2\}\}$. We have $\Lambda^{+}(\mathscr{B}) \stackrel{\mathcal{N}}{=}\{\beta\}$ while $\Lambda^{+}(\mathcal{C})=\{a \beta+(1-a) \gamma \mid a \in(0,1)\}$.

Now, for a vector $\beta \in \mathbb{R}^{\mathcal{N}}$, we define the set

$V(\beta)=\left\{v \in \mathrm{TU}^{N} \mid \sum_{S \in \mathcal{N}} \beta_{S} v(S) \leq v(N)\right\}$

of transferable utility games for which the weighted sum of the values of the coalitions with respect to $\beta$ is less than or equal to the worth of the grand coalition. Also, we define $V^{+}(\mathscr{B})=$ $\cap_{\beta \in \Lambda^{+}(\mathcal{B})} V(\beta)$ and $V^{+}=\cap_{\mathscr{B} \in \mathbb{B}^{N}} V^{+}(\mathscr{B})$. So, $V^{+}(\mathscr{B})$ is the set of games that satisfy the constraints imposed by all balanced weight vectors for collection $\mathscr{B}$, and $V^{+}$is the set of games that satisfy the constraints imposed by all balanced weight vectors.

Consider some $\mathscr{B} \in \mathbb{B}^{N}$. Note that $v \in V(\beta)$ for some $\beta \in$ $\Lambda^{+}(\mathscr{B})$ does not imply that $v \in V^{+}(\mathscr{B})$. This is illustrated by the following example.

Example 2.3. Consider a three person game $v \in \mathrm{TU}^{N}$ such that $v(\{1\})=2, v(\{1,2\})=8, v(\{1,3\})=8, v(\{2,3\})=4$ and $v(N)=8$. We find that the balanced collection $\mathcal{B}=\{\{1\},\{1,2\}$, $\{1,3\},\{2,3\}\}$ corresponds with more than one balanced weight vector, for instance $\beta=\left(\frac{1}{2}, \frac{1}{4}, \frac{1}{4}, \frac{3}{4}\right)$ and $\gamma=\left(\frac{1}{4}, \frac{3}{8}, \frac{3}{8}, \frac{5}{8}\right)$. We have that

$$
\begin{aligned}
\sum_{S \in \mathcal{B}} \beta_{S} v(S) & =\frac{1}{2} v(\{1\})+\frac{1}{4} v(\{1,2\})+\frac{1}{4} v(\{1,3\})+\frac{3}{4} v(\{2,3\}) \\
& =8=v(N),
\end{aligned}
$$

but

$$
\begin{aligned}
\sum_{S \in \mathcal{B}} \gamma_{S} v(S) & =\frac{1}{4} v(\{1\})+\frac{3}{8} v(\{1,2\})+\frac{3}{8} v(\{1,3\})+\frac{5}{8} v(\{2,3\}) \\
& =9>v(N) .
\end{aligned}
$$

So, $v \in V(\beta)$ but $v \notin V(\gamma)$. This implies that $v \notin V^{+}(\mathcal{B})$. 
We call a game $v \in \mathrm{TU}^{N}$ balanced if $v \in V^{+}$.

Theorem 2.4 (Bondareva, 1963; Shapley, 1967). Let $v \in \mathrm{TU}^{N}$. Then $C(v) \neq \varnothing$ if and only if $v \in V^{+}$.

It is well known that not all balanced collections are necessary to guarantee that a game is balanced. Minimal balanced collections suffice to characterize the class of games with a non-empty core.

Definition 2.5. A collection $\mathscr{B} \in \mathbb{B}^{N}$ is called minimal balanced if there does not exist a $C \subset \mathscr{B}$ such that $\mathcal{C} \in \mathbb{B}^{N}$. The class of minimal balanced collections on player set $N$ is denoted by $\mathbb{B}_{\min }^{N}$

Note that in Example 2.2, only the collection $\{\{1\},\{2\}\}$ is minimal balanced. We define $V_{\min }^{+}=\bigcap_{\mathscr{B} \in \mathbb{B}_{\min }^{N}} V^{+}(\mathscr{B})$ as the class of games that satisfy the constraints originating from minimal balanced collections.

Theorem 2.6 (Bondareva, 1963; Shapley, 1967). A game $v \in \mathrm{TU}^{N}$ is balanced if and only if $v \in V_{\mathrm{min}}^{+}$, i.e., $V^{+}=V_{\mathrm{min}}^{+}$.

Not only do we need just the minimal balanced collections to characterize the non-emptiness of the core, an additional advantage of minimal balanced collections is that for every minimal balanced collection there exists only one balanced vector of weights. For the following theorem, we provide the proof by Peleg and Sudhölter (2003) as we will use a similar technique later on to prove results on minimal exact balanced collections.

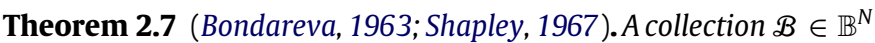
is minimal balanced if and only if $\left|\Lambda^{+}(\mathcal{B})\right|=1$.

Proof (Peleg and Sudhölter, 2003). Let $\mathscr{B} \in \mathbb{B}^{N}$. Take $\beta \in \Lambda^{+}(\mathscr{B})$.

First we show that a balanced collection that is not minimal corresponds to more than one balanced weight vector. If $\mathcal{C} \subsetneq \mathscr{B}$ is a balanced collection with weights $\gamma \in \Lambda^{+}(\mathcal{C})$, then it is readily verified that $a \gamma+(1-a) \beta \in \Lambda^{+}(\mathscr{B})$ for $a \in[0,1)$, so the weight vector for $B$ is not unique.

Second, we show that every collection with more than one balanced weight vector is not minimal. Assume that there exists another weight vector $\alpha \in \Lambda(\mathscr{B}), \alpha \neq \beta$. As there exists a coalition $S \in \mathscr{B}$ such that $\beta_{S}>\alpha_{S}$, we obtain that $a=\min \left\{\frac{\alpha_{S}}{\beta_{S}-\alpha_{S}} \mid\right.$ $\left.\beta_{S}>\alpha_{S}\right\}$ is well defined. Let $\gamma_{S}=(1+a) \alpha_{S}-a \beta_{S}$ for all $S \in \mathscr{B}$. Then $\mathcal{C}=\left\{S \in \mathscr{B} \mid \gamma_{S}>0\right\}$ is a proper subcollection of $\mathscr{B}$ with $\gamma \in \Lambda^{+}(\mathcal{C})$. So, $\mathcal{C} \in \mathbb{B}^{N}$ and $\mathbb{B}$ is not minimal.

The following theorem states that we cannot characterize the set of balanced games by a subset of the minimal balanced collections.

Theorem 2.8 (Bondareva, 1963; Shapley, 1967). Let $\mathcal{B} \in \mathbb{B}_{\min }^{N}$. Then there exists a game $v \in \mathrm{TU}^{N}$ such that $v \in V^{+}(\mathcal{C})$ for all collections $\mathcal{C} \in \mathbb{B}_{\min }^{N} \backslash\{\mathscr{B}\}$ and $v \notin V^{+}(\mathscr{B})$.

\section{Exact balancedness}

Games with a non-empty core can be characterized using balanced collections. A similar characterization exists for exact games. Exact games form a subclass of the class of games with a non-empty core.

Definition 3.1. A game $v \in \mathrm{TU}^{N}$ is exact if for every coalition $S \in \mathcal{N}$ there exists an $x \in C(v)$ such that $\sum_{i \in S} x_{i}=v(S)$.

Schmeidler (1972) provides a characterization of exact games. Csóka et al. (2011) introduces two different characterizations of exact games, one using total balancedness and overbalancedness, and one using exact balancedness. For a discussion on these three characterizations we refer to Csóka et al. (2011). Here, we use exact balancedness as defined by Csóka et al. (2011) except that in line with Definition 2.1 we exclude the trivial collection $\{N\}$.
Definition 3.2 (Csóka et al., 2011). For a collection $\mathcal{E} \subseteq \mathcal{N}, \mathcal{E} \neq$ $\{N\}$, a vector of weights $\lambda \in \mathbb{R}^{\mathcal{N}}$ is called exact balanced if there exists a $T \in \mathcal{E}$ such that $\lambda_{S}>0$ for all $S \in \mathscr{E} \backslash\{T\}, \lambda_{T} \neq 0, \lambda_{S}=0$ for all $S \notin \varepsilon$, and $\sum_{S \in \mathcal{E}} \lambda_{S} e^{S}=e^{N}$. We denote $\Lambda(\mathcal{E})$ for the set of all exact balanced vectors on $\mathcal{E}$. A collection $\mathscr{E} \subseteq \mathcal{N}$ is called exact balanced if $\Lambda(\mathscr{E}) \neq \emptyset$. Denote $\mathbb{E}^{N}$ for the set of all exact balanced collections on player set $N$, and $\Lambda=\cup_{\mathcal{E} \in \mathbb{E}^{N}} \Lambda(\mathcal{E})$.

In the remainder, we will typically use $\varepsilon$ and $\mathscr{D}$ to denote exact balanced collections, and use $\lambda$ and $\delta$ to denote their respective weight vectors.

Note the discrepancy with the definition of balanced vectors. For exact balanced weight vectors, we allow for one negative weight. As Csóka et al. (2011) argues, the negative weight in an exact balanced collection can be interpreted as players in the coalition with a negative weight working overtime in other coalitions, and paying the opportunity cost of doing so to the coalition that is active for a negative amount of time. It is readily checked that $\Lambda^{+}(\mathcal{E}) \subseteq \Lambda(\mathcal{E})$ for every $\mathscr{E} \subseteq \mathcal{N}$, and therefore $\mathbb{B}^{N} \subseteq \mathbb{E}^{N}$. In contrast with $\Lambda^{+}, \Lambda$ is not a convex set in general, since a convex combination of two elements of $\Lambda$ is not necessarily an element of $\Lambda$.

Example 3.3. Let $N=\{1,2,3\}$. Take $\lambda, \delta \in \mathbb{R}^{\mathcal{N}}$ such that $\lambda_{\{1,2\}}=$ $\lambda_{\{1,3\}}=1, \lambda_{\{1\}}=-1$ and $\delta_{\{1,2\}}=\delta_{\{2,3\}}=1, \delta_{\{2\}}=-1$. Clearly, $\lambda$ and $\delta$ are exact balanced weight vectors. However, the convex combination $\frac{1}{2}(\lambda+\delta)$ is not an exact balanced weight vector, as it has two negative components. This means that $\Lambda$ is not a convex set.

Define, similar to the definitions of $V^{+}(\mathscr{B})$ and $V^{+}, V(\mathscr{E})=$ $\cap_{\lambda \in \Lambda(\mathcal{E})} V(\lambda)$ for all $\mathscr{E} \in \mathbb{E}^{N}$ and $V=\cap_{\mathscr{E} \in \mathbb{E}^{N}} V(\mathscr{E})$. As $V(\lambda)$ is the class of games that satisfy the constraint imposed by weight vector $\lambda, V(\mathscr{E})$ is the set of all games that satisfy the constraints imposed by the exact balanced weight vectors of the collection $\&$ and $V$ is the class of exact balanced games.

Theorem 3.4 (Csóka et al., 2011). A game $v \in \mathrm{TU}^{N}$ is exact if and only if $v \in V$.

So, just as balancedness is equivalent with non-emptiness of the core we have that exact balancedness is equivalent with the existence for every coalition of a core element where this coalition gets precisely its stand-alone value. Similar to the definition of minimal balanced collections, we define minimal exact balanced collections.

Definition 3.5. A collection $\mathcal{E} \in \mathbb{E}^{N}$ is minimal exact balanced if there exists no $\mathscr{D} \subsetneq \mathcal{E}$ such that $\mathscr{D} \in \mathbb{E}^{N}$. We denote $\mathbb{E}_{\text {min }}^{N}$ for the class of minimal exact balanced collections.

Example 3.6. Regarding exact balancedness, a similar reasoning as in Example 2.2 can be used to show that only $\{\{1\},\{2\}\}$ and $\{\{1\},\{2\},\{1,2\}\}$ are exact balanced for two-person games. Since clearly $\Lambda(\mathcal{E})=\Lambda^{+}(\mathscr{E})$ for every $\& \in \mathbb{E}^{N}$, we have $\mathbb{E}^{N}=\mathbb{B}^{N}$ and $\mathbb{E}_{\text {min }}^{N}=\mathbb{B}_{\min }^{N}$. This is not surprising, since for two-player games, whenever the core is non-empty there exists a core element where player 1 gets $v(\{1\})$ and there exists a core element where player 2 gets $v(\{2\})$. So, the concepts of balancedness and exactness are equivalent for two player games. For games with three or more players, $\mathbb{B}^{N} \subsetneq \mathbb{E}^{N}$. For a player set consisting of three players, $\mathbb{E}_{\text {min }}^{N}$ and $\mathbb{B}_{\min }^{N}$ are given in Table 1.

If the size of the player set increases, the number of collections in the different classes grows considerably. Table 2 shows the number of collections in all classes for up to 4 players. The minimal balanced collections as well as the minimal exact balanced 
Table 1

Minimal balanced and minimal exact balanced collections for $N=\{1,2,3\}$.

$\mathbb{B}_{\min }^{N} \quad \mathbb{E}_{\text {min }}^{N}$

$\{1\},\{2\},\{3\}$

$\{1\},\{2\},\{3\}$

$\{1,2\},\{3\}$

$\{1,2\},\{3\}$

$\{1,3\},\{2\}$

$\{2,3\},\{1\}$

$\{1,3\},\{2\}$

$\{1,2\},\{1,3\},\{2,3\}$

$\{2,3\},\{1\}$

$\{1,2\},\{1,3\},\{2,3\}$

$\{1\},\{1,2\},\{1,3\}$

$\{2\},\{1,2\},\{2,3\}$

$\{3\},\{1,3\},\{2,3\}$

$\{1\},\{2\},\{1,2\}, N$

$\{1\},\{3\},\{1,3\}, N$

$\{2\},\{3\},\{2,3\}, N$

Table 2

Number of collections in different classes.

\begin{tabular}{lrr}
\hline$|N|$ & 3 & \multicolumn{1}{c}{4} \\
\hline$\left|\mathbb{B}^{N}\right|$ & 42 & 18878 \\
$\left|\mathbb{B}_{\min }^{N}\right|$ & 5 & 41 \\
$\left|\mathbb{E}^{N}\right|$ & 63 & 27014 \\
$\left|\mathbb{E}_{\min }^{N}\right|$ & 11 & 165 \\
\hline
\end{tabular}

collections are generated using methods introduced later on in this paper.

As we have shown in Theorem 2.7, the class of minimal balanced collections coincides with the set of balanced collections with a unique weight vector. For minimal exact balanced collections, a somewhat weaker statement holds: the class of minimal exact balanced collections not containing the grand coalition coincides with the set of exact balanced collections with a unique weight vector.

Theorem 3.7. Let $\varepsilon \in \mathbb{E}^{N}$. Then $\varepsilon \in \mathbb{E}_{\min }^{N}$ and $N \notin \mathcal{E}$ if and only if $|\Lambda(\mathcal{E})|=1$.

Proof. We prove the 'only if' part of the theorem by showing that we can construct an exact balanced subcollection of $\varepsilon$ if the weight vector is not unique. Take $\varepsilon \in \mathbb{E}_{\min }^{N}$ with $N \notin \varepsilon$. Suppose that there exist two weight vectors $\lambda, \mu \in \Lambda(\mathscr{E})$ such that $\lambda \neq \mu$.

If both $\lambda \in \Lambda^{+}(\mathscr{E})$ and $\mu \in \Lambda^{+}(\mathcal{E})$, we have by Theorem 2.7 that $\varepsilon \notin \mathbb{B}_{\min }^{N}$. Hence, there exists an exact balanced subcollection of $\mathcal{E}$ in this case.

Next assume $\& \in \mathbb{B}_{\min }^{N}, \lambda \in \Lambda^{+}(\mathcal{E})$ and $\mu \notin \Lambda^{+}(\mathcal{E})$. Let $U \in \mathcal{E}$ be such that $\mu_{U}<0$, and take $a=\min \left\{\frac{\lambda_{S}}{\mu_{S}} \mid S \in \mathcal{E} \backslash\{U\}\right\}$ and $\beta=\frac{1}{1-a}(\lambda-a \mu)$. Note that $0<a<1$ since $\lambda_{S}>0$ and $\mu_{S}>0$ for all $S \in \mathcal{E} \backslash\{U\}$, and $a \geq 1$ would imply that

$$
\begin{aligned}
e^{N} & =\sum_{S \in \mathcal{E}} \mu_{S} e^{S}=\sum_{S \in \mathcal{E} \backslash\{U\}} \mu_{S} e^{S}+\mu_{U} e^{U}<\sum_{S \in \mathcal{E} \backslash\{U\}} \lambda_{S} e^{S}+\lambda_{U} e^{U} \\
& =\sum_{S \in \mathcal{E}} \lambda_{S} e^{S} \leq e^{N}
\end{aligned}
$$

where the strict inequality uses that $\mu_{U}<0<\lambda_{U}$. Note that $\beta_{S}=\frac{1}{1-a}\left(\lambda_{S}-a \mu_{S}\right) \geq 0$ for all $S \in \mathcal{E}$, with equality for at least one coalition. If we take $\mathscr{B}=\left\{S \in \mathcal{E} \mid \beta_{S}>0\right\}$, then $\mathscr{B}$ is a proper subset of $\mathcal{E}$ and $\sum_{S \in \mathscr{B}} \beta_{S} e^{S}=\sum_{S \in \mathcal{E}} \beta_{S} e^{S}=$ $\sum_{S \in \mathcal{E}}\left(\frac{\lambda S}{1-a} e^{S}-\frac{a \mu_{S}}{1-a} e^{S}\right)=e^{N}$, so $\mathscr{B} \in \mathbb{B}^{N}$ which contradicts $\varepsilon \in \mathbb{B}_{\text {min }}^{N}$.

Finally, let $\lambda \notin \Lambda^{+}(\mathcal{E})$ and $\mu \notin \Lambda^{+}(\mathcal{E})$. This means that there exist coalitions $T \in \mathcal{E}$ and $U \in \mathcal{E}$ such that $\lambda_{T}<0$ and $\mu_{U}<0$.

Assume $T=U$. Take $a=\min \left\{\frac{\lambda_{S}}{\mu_{S}} \mid S \in \mathcal{E}\right\}$. Note that $a>0$ since for $S \in \mathcal{E}$ either both $\lambda_{S}>0$ and $\mu_{S}>0$ or both $\lambda_{S}<0$ and $\mu_{S}<0$. It holds that $a<1$, as $a \geq 1$ would imply that either $\lambda=\mu$ or $\sum_{S \ni i} \lambda_{S}>\sum_{S \ni i} \mu_{S}=1$ for $i \in N \backslash T$, a non-empty set since by assumption $N \notin \mathcal{E}$. We construct $\delta_{S}=\frac{1}{1-a} \lambda_{S}-\frac{a}{1-a} \mu_{S}$ for all $S \in \mathcal{E}$ and $\mathscr{D}=\left\{S \in \mathcal{E} \mid \delta_{S} \neq 0\right\}$. It is readily verified that $\delta_{S} \geq 0$ for all $S \in \mathscr{D} \backslash\{T\}$ and $\sum_{S \in \mathscr{D}} \delta_{S} e^{S}=e^{N}$. This shows that $\mathscr{D} \in \mathbb{E}^{N}$ and by construction $\mathscr{D} \subsetneq \mathcal{E}$, which contradicts $\mathscr{E} \in \mathbb{E}_{\min }^{N}$.

Now assume $T \neq U$. Take $a=\frac{\mu_{T}}{\mu_{T}-\lambda_{T}}$. It is readily checked that $0<a<1$. Take $\delta=a \lambda+(1-a) \mu$ and $\mathscr{D}=\left\{S \in \mathcal{E} \mid \delta_{S} \neq 0\right\}$. We have $\delta_{S}>0$ for every $S \in \mathscr{D} \backslash\{T, U\}$ and $\delta_{T}=0$. Since $\sum_{S \in \mathcal{D}} \delta_{S} e^{S}=\sum_{S \in \mathcal{E}} a \lambda_{S} e^{S}+\sum_{S \in \mathcal{E}}(1-a) \mu_{S} e^{S}=e^{N}$ this shows that $\mathscr{D} \in \mathbb{E}^{N}$ which contradicts $\& \in \mathbb{E}_{\min }^{N}$.

To prove the 'if' part of the theorem, let $\mathcal{E} \subseteq \mathcal{N}$ be such that $\Lambda(\mathcal{E})=\{\lambda\}$ for some $\lambda \in \mathbb{R}^{\mathcal{N}}$. First suppose $\varepsilon \notin \mathbb{E}_{\min }^{N}$. We show that we can construct a second weight vector in $\Lambda(\mathscr{E})$. As $\mathcal{E} \notin \mathbb{E}_{\text {min }}^{N}$, there exists an exact balanced subcollection $\mathscr{D} \subsetneq \mathcal{E}$. Take $\mu \in \Lambda(\mathscr{D})$ and define the function $f:[0,1] \rightarrow \mathbb{R}^{\stackrel{*}{\mathcal{N}}}$ by $f(b)=(1-b) \lambda+b \mu$. As $f$ is continuous, there exists an $\epsilon>0$ such that the sign of $f_{S}(\epsilon)$ coincides with the sign of $\lambda_{S}$ for all $S \in \varepsilon$. Since $\sum_{S \in \mathcal{E}} f_{S}(\epsilon) e^{S}=\sum_{S \in \mathcal{E}}(1-\epsilon) \lambda_{S} e^{S}+\sum_{S \in \mathcal{D}} \epsilon \mu_{S} e^{S}=e^{N}$, we obtain that $f(\epsilon) \in \Lambda(\mathcal{E})$ while $f(\epsilon) \neq \lambda$, a contradiction.

Secondly, suppose $N \in \mathcal{E}$. It is readily checked that $\lambda_{N} \leq 1$, and if $\lambda_{N}<1$ we obtain that the collection $\mathcal{A}=\mathscr{E} \backslash\{N\}$ is exact balanced with weight vector $\mu_{S}=\frac{\lambda_{S}}{1-\lambda_{N}}$ for every $S \in \mathcal{A}$ which contradicts $\varepsilon \in \mathbb{E}_{\text {min }}^{N}$. Hence, $\lambda_{N}=1$. As $\sum_{S \in \mathcal{E} \backslash\{N\}} \lambda_{S} e^{S}=0$, we have that $\sum_{S \in \mathcal{E} \backslash\{N\}} 2 \lambda_{S} e^{S}=0$. Define the weight vector $\mu$ by $\mu_{S}=2 \lambda_{S}$ for all $S \in \mathcal{E} \backslash\{N\}, \mu_{N}=1$ and $\mu_{S}=0$ otherwise. It is readily checked that $\mu \in \Lambda(\varepsilon)$ with $\mu \neq \lambda$, a contradiction.

There exist minimal exact balanced collections with more than one exact balanced weight vector. By Theorem 3.7 such a collection must contain the set $N$.

Example 3.8. Take $N=\{1,2,3\}$. The collection $\&=\{\{1\},\{2\}$, $\{1,2\}, N\}$ is minimal exact balanced, but there exists more than one weight vector: define $\lambda$ by $\lambda_{\{1\}}=\lambda_{\{2\}}=1, \lambda_{\{1,2\}}=-1$ and $\lambda_{N}=1$ and $\mu$ by $\mu_{\{1\}}=\mu_{\{2\}}=2, \mu_{\{1,2\}}=-2$ and $\mu_{N}=1$. It is readily checked that $\lambda \in \Lambda(\mathcal{E})$ and $\mu \in \Lambda(\mathscr{E})$.

If a minimal exact balanced collection does contain the grand coalition, then there exists more than one exact balanced weight vector, but these weight vectors are related in a special way and induce the same constraint on the game. Furthermore, if for an exact balanced collection all weight vectors induce the same constraint on the game, then the collection is minimal exact balanced.

Theorem 3.9. Let $\& \in \mathbb{E}^{N}$. Then $\& \in \mathbb{E}_{\text {min }}^{N}$ and $N \in \mathcal{E}$ if and only if for every $\lambda \in \Lambda(\&)$ and $\mu \in \Lambda(\mathscr{E})$ there exists a scalar $a>0$ such that

$\mu_{S}=a \lambda_{S} \quad$ for all $S \in \mathcal{E} \backslash\{N\}$,

$\mu_{N}=\lambda_{N}=1$.

Proof. For the 'only if' part of the proof, let $\varepsilon \in \mathbb{E}_{\text {min }}^{N}$ be such that $N \in \mathscr{E}$. Let $\lambda \in \Lambda(\mathscr{E})$. It is readily checked that $\lambda_{N} \leq 1$, and if $\lambda_{N}<1$ we obtain that the collection $\mathcal{C}=\mathcal{E} \backslash\{N\}$ is exact balanced with weight vector $\gamma_{S}=\frac{\lambda_{S}}{1-\lambda_{N}}$ for every $S \in \mathcal{C}$. Hence, $\lambda_{N}=1$.

Take $T \in \mathcal{E}$ such that $\lambda_{T}<0$. Such an $T \in \mathcal{E}$ exists, as $N \in \mathcal{E}$ and therefore $\mathcal{E} \notin \mathbb{B}_{\min }^{N}$. As $\sum_{S \in \mathcal{E} \backslash\{N\}} \lambda_{S} e^{S}=0$ and $\lambda_{S}>0$ for all $S \in \mathcal{E} \backslash\{T\}$, we obtain that $S \subsetneq T$ for all $S \in \mathcal{E} \backslash\{T, N\}$. This implies that the location of the negative weight is unique, $\mu_{T}<0$ for every $\mu \in \Lambda(\mathcal{E})$. Rewriting $\sum_{S \in \mathcal{E} \backslash\{N\}} \lambda_{S} e^{S}=0$ yields $\sum_{S \in \mathscr{E} \backslash\{N\}}-\frac{\lambda_{S}}{\lambda_{T}} e^{S}=e^{T}$, and therefore $\mathcal{E} \backslash\{N, T\} \in \mathbb{B}^{T}$. If there exists a minimal balanced collection $\mathscr{B} \in \mathbb{B}_{\min }^{T}$ such that $\mathscr{B} \subsetneq \mathcal{E} \backslash\{T, N\}$, it is readily checked that $\mathcal{B} \cup\{T, N\}$ is an exact balanced collection, which contradicts our assumption of $\mathscr{E} \in \mathbb{E}_{\min }^{N}$. Hence, $\mathscr{E} \backslash\{N, T\} \in \mathbb{B}_{\min }^{T}$. Since $\mathcal{E} \backslash\{N, T\} \in \mathbb{B}_{\min }^{T}$, by Theorem 2.7 
there is a unique balanced vector of weights $\beta$ of $\mathcal{E} \backslash\{N, T\}$. Note that

$$
\begin{aligned}
e^{N} & =e^{N}+\lambda_{T} e^{T}+\sum_{S \in \mathcal{E} \backslash\{N, T\}} \lambda_{S} e^{S} \\
& =e^{N}+\lambda_{T} \sum_{S \in \mathcal{E} \backslash\{N, T\}} \beta_{S} e^{S}+\sum_{S \in \mathcal{E} \backslash\{N, T\}} \lambda_{S} e^{S} \\
& =e^{N}+\sum_{S \in \mathcal{E} \backslash\{N, T\}}\left(\lambda_{T} \beta_{S}+\lambda_{S}\right) e^{S} .
\end{aligned}
$$

This implies that $\sum_{S \in \mathcal{E} \backslash\{N, T\}}\left(\lambda_{T} \beta_{S}+\lambda_{S}\right) e^{S}=0$. If $\lambda_{T} \beta_{S} \neq \lambda_{S}$ for some $S \in \mathcal{E} \backslash\{N, T\}$ we have $\beta+\epsilon\left(\lambda_{T} \beta+\lambda\right) \in \mathbb{B}_{\min }^{T}$ for small $\epsilon>0$. So, $\lambda_{T} \beta_{S}+\lambda_{S}=0$ and therefore $\lambda_{S}=-\lambda_{T} \beta_{S}$ for every $S \in \mathscr{E} \backslash\{N, T\}$. Now take $\mu \in \Lambda(\mathcal{E})$ and take $a=\frac{\mu_{T}}{\lambda_{T}}$. Since $\mu_{T}<0$ and $\lambda_{T}<0, a>0$. We have $\mu_{T}=a \lambda_{T}$ by definition, and $\mu_{S}=-\mu_{T} \beta_{S}=-a \lambda_{T} \beta_{S}=\lambda_{S}$ for every $S \in \mathcal{E} \backslash\{N, T\}$.

For the 'if' part of the proof, clearly $N \in \mathcal{E}$. Suppose $\& \notin \mathbb{E}_{\mathrm{min}}^{N}$ As $\mathscr{E}$ is not minimal, there exists a $\mathscr{D} \subsetneq \mathcal{E}$ such that $\mathscr{D} \in \mathbb{E}_{\min }^{N}$. Let $\lambda \in \Lambda(\mathscr{E})$ and $\delta \in \Lambda(D)$. Define $\mu=(1-b) \lambda+b \delta$, where $b>0$ is sufficiently small, such that the sign of $\delta_{S}$ equals the sign of $\mu_{S}$ for every $S \in \mathcal{E}$. Clearly, $\mu \in \Lambda(\mathcal{E})$. Take $T \in \mathcal{E} \backslash \mathbb{D}$ and $U \in \mathscr{E} \cap \mathscr{D}, U \neq N$. Such a $U$ exists, as $\{N\}$ is not a minimal exact balanced collection by definition. Since $\mu_{T}=(1-b) \lambda_{T}$ and $\mu_{U}=(1-b) \lambda_{U}+b \delta_{U} \neq(1-b) \lambda_{U}$, there does not exist a scalar $a>0$ such that $\mu_{T}=a \lambda_{T}$ and $\mu_{U}=a \lambda_{U}$, which is a contradiction.

We have shown that for minimal exact balanced collections either the corresponding weight vector is unique or all corresponding weight vectors induce the same constraint on the game. This enables us to use one standardized weight vector for every minimal exact balanced collection. In the remainder, for every minimal balanced collection $\mathscr{B}$ we denote $\beta^{\mathcal{B}}$ for the unique balanced weight vector. More general, for every $\varepsilon \in \mathbb{E}_{\text {min }}^{N}$ with $N \notin \mathcal{E}$, we denote $\lambda^{\varepsilon}$ for the unique exact balanced weight vector. For $\varepsilon \in \mathbb{E}_{\min }^{N}$ with $N \in \mathcal{E}, \lambda^{\mathcal{E}}$ denotes the unique standardized exact balanced weight vector such that $\min \left\{\lambda_{S}^{\mathcal{E}} \mid S \in \mathcal{E}\right\}=-1$. Notice that for notational convenience, for $\mathscr{B} \in \mathbb{B}_{\min }^{N}$ the standardized weight vector is both denoted by $\beta^{\mathscr{B}}$ and $\lambda^{\mathscr{B}}$.

\section{Partitioning the class of minimal exact balanced collections}

In this section we study the structure of the class of minimal exact balanced collections. It turns out that this set can be decomposed in three parts, all related to balanced collections. The first part consists of all minimal balanced collections.

Theorem 4.1. $\mathbb{B}_{\min }^{N} \subseteq \mathbb{E}_{\min }^{N}$.

Proof. Let $\mathscr{B} \in \mathbb{B}_{\min }^{N}$. It is clear that every minimal balanced collection is also exact balanced. It remains to show that it is also minimal exact balanced. Assume there exists an exact balanced collection $\mathcal{E} \subsetneq \mathscr{B}$ and take $\lambda \in \Lambda(\mathcal{E})$. We will show that this results in a contradiction with $\mathcal{B} \in \mathbb{B}_{\text {min }}^{N}$.

Since $\mathcal{B} \in \mathbb{B}_{\min }^{N}$ we know that there exists a $T \in \mathscr{E}$ such that $\lambda_{T}<0$ as $\mathcal{B}$ does not have a proper subset that is balanced. Take $a=\min \left\{\frac{\beta_{S}^{\mathscr{B}}}{\lambda_{S}} \mid S \in \mathcal{E} \backslash\{T\}\right\}$ and $\gamma=\frac{1}{1-a}\left(\beta^{\mathscr{B}}-a \lambda\right)$. Note that $0<a<1$ since $\beta_{S}^{\mathscr{B}}>0$ and $\lambda_{S}>0$ for all $S \in \mathcal{E} \backslash\{T\}$, and $a \geq 1$ would imply that

$$
\begin{aligned}
\sum_{S \in \mathcal{E}} \lambda_{S} e^{S} & =\sum_{S \in \mathcal{E} \backslash\{T\}} \lambda_{S} e^{S}+\lambda_{T} e^{T}, \\
& <\sum_{S \in \mathcal{E} \backslash\{T\}} \beta_{S}^{\mathcal{B}} e^{S}+\beta_{T}^{\mathcal{B}} e^{T}, \\
& =\sum_{S \in \mathcal{E}} \beta_{S}^{\mathcal{B}} e^{S}, \\
& \leq e^{N} .
\end{aligned}
$$

Now $\gamma_{S} \geq 0$ for all $S \in \mathscr{B}$, with equality for at least one coalition. Take $\mathcal{C}=\left\{S \in \mathcal{E} \mid \gamma_{S}>0\right\}$. Then $\mathcal{C}$ is a proper subset of $\mathscr{B}$ and

$$
\begin{aligned}
\sum_{S \in \mathcal{C}} \gamma_{S} e^{S} & =\sum_{S \in \mathscr{B}} \gamma_{S} e^{S}=\sum_{S \in \mathscr{B}} \frac{\beta_{S}^{\mathcal{B}}}{1-a} e^{S}-\sum_{S \in \mathcal{E}} \frac{a \lambda_{S}}{1-a} e^{S} \\
& =\frac{1}{1-a} e^{N}-\frac{a}{1-a} e^{N}=e^{N},
\end{aligned}
$$

so $\mathcal{C} \in \mathbb{B}^{N}$, contradicting $\mathscr{B} \in \mathbb{B}_{\text {min }}^{N}$.

The second part of the partition of $\mathbb{E}_{\text {min }}^{N}$ consists of so-called negative balanced collections. The set of all negative balanced collections is denoted by $\overline{\mathbb{B}}_{\text {min }}^{N}$. The negative balanced collections can be obtained, by replacing one coalition in a minimal balanced collection by its complement. However, this is only allowed for the coalitions with weight strictly smaller than 1 . We have

$$
\overline{\mathbb{B}}_{\text {min }}^{N}=\left\{(\mathscr{B} \backslash\{S\}) \cup(\{N \backslash S\}) \mid \mathscr{B} \in \mathbb{B}_{\min }^{N}, S \in \mathscr{B}: \beta_{S}^{\mathcal{B}}<1\right\} .
$$

Example 4.2. Let $N=\{1,2,3,4\}$, and consider the minimal balanced collection $\mathscr{B}=\{\{1,2\},\{1,3\},\{2,3\},\{4\}\}$. For the weight vector $\beta^{\mathscr{B}}$ it holds that $\beta_{\{1,2\}}^{\mathscr{B}}=\frac{1}{2}$. This means that $\mathcal{E}=(\mathscr{B} \backslash$ $\{\{1,2\}\}) \cup(\{\{3,4\}\})=\{\{3,4\},\{1,3\},\{2,3\},\{4\}\} \in \overline{\mathbb{B}}_{\min }^{N}$. It is readily checked that $\mathscr{E} \in \mathbb{E}^{N}$, since $e^{\{1,3\}}+e^{\{2,3\}}+2 e^{\{4\}}-e^{\{3,4\}}=e^{N}$. As $\beta_{\{4\}}^{\mathcal{B}}=1$, we cannot replace the coalition $\{4\}$ by its complement to obtain an element of $\overline{\mathbb{B}}_{\text {min }}^{N}$.

For every negative balanced collection we use the balanced weight vector of the corresponding minimal balanced collection to compute an exact balanced weight vector. The negative weight of the exact balanced weight vector is placed on the coalition that has replaced its complement.

Theorem 4.3. Let $\& \in \overline{\mathbb{B}}_{\min }^{N}$. Let $\mathcal{B} \in \mathbb{B}_{\min }^{N}$ and $U \in \mathscr{B}$ be such that $\mathcal{E}=(\mathscr{B} \backslash\{U\}) \cup(\{N \backslash U\})$. Let $\lambda_{S}=\frac{\beta_{S}^{B}}{1-\beta_{U}^{B}}$ for all $S \in \mathscr{B} \backslash\{U\}, \lambda_{N \backslash U}=$ $-\frac{\beta_{U}^{\mathscr{B}}}{1-\beta_{U}^{\mathcal{B}}}$ and $\lambda_{S}=0$ for $S \in \mathcal{N} \backslash \mathcal{E}$. Then $\lambda \in \Lambda(\mathcal{E})$.

Proof. As $\mathscr{B} \in \mathbb{B}_{\min }^{N}$ and $\beta_{U}^{\mathscr{B}}<1$, we know $N \backslash U \notin \mathscr{B}$. As $0<\beta_{U}^{\mathscr{B}}<1$, we obtain that $\lambda_{S}=\frac{\beta_{S}^{\mathscr{B}}}{1-\beta_{U}^{B}}>0$ for all $S \in \mathcal{E} \backslash\{U\}$ and $\lambda_{N \backslash U}=-\frac{\beta_{U}^{\mathscr{B}}}{1-\beta_{U}^{\mathcal{B}}}<0$. For $i \in U$,

$$
\sum_{S \in \mathcal{E}, S \ni i} \lambda_{S}=\sum_{S \in \mathcal{B} \backslash\{U\}, S \ni i} \frac{\beta_{S}^{\mathcal{B}}}{1-\beta_{U}^{\mathcal{B}}}=\frac{1}{1-\beta_{U}^{\mathcal{B}}} \sum_{S \in \mathcal{B} \backslash\{U\}, S \ni i} \beta_{S}^{\mathcal{B}}=1
$$

and for $i \in N \backslash U$ it holds that

$$
\sum_{S \in \mathcal{E}, S \ni i} \lambda_{S}=\sum_{S \in \mathscr{B} \backslash\{U\}, S \ni i} \frac{\beta_{S}^{\mathcal{B}}}{1-\beta_{U}^{\mathcal{B}}}-\frac{\beta_{U}^{\mathcal{B}}}{1-\beta_{U}^{\mathcal{B}}}=1 .
$$

So, indeed $\lambda \in \Lambda(\mathcal{E})$.

By definition of $\overline{\mathbb{B}}_{\text {min }}^{N}$ and the observation that $N \notin \mathscr{B}$ for every $\mathscr{B} \in \mathbb{B}_{\text {min }}^{N}$, we have $N \notin \mathcal{E}$ for every $\mathcal{E} \in \overline{\mathbb{B}}_{\min }^{N}$. Hence, for this second part of the partition we can focus on collections without the grand coalition. Consider such a collection which is not minimal balanced. Then it is minimal exact balanced if and only if it is negative balanced. This also implies that the exact balanced weight vector of Theorem 4.3 is in fact the unique exact balanced weight vector.

\section{Theorem 4.4.}

(i) $\overline{\mathbb{B}}_{\min }^{N} \subseteq \mathbb{E}_{\min }^{N} \backslash \mathbb{B}_{\min }^{N}$.

(ii) Let $\& \in \mathbb{E}_{\min }^{N} \backslash \mathbb{B}_{\text {min }}^{N}$ and $N \notin \mathcal{E}$. Then $\varepsilon \in \overline{\mathbb{B}}_{\text {min }}^{N}$. 
Proof of (i). Let $\mathcal{E} \in \overline{\mathbb{B}}_{\min }^{N}$. Let $\mathscr{B} \in \mathbb{B}_{\min }^{N}$ and $U \in \mathscr{B}$ be such that $\mathcal{E}=(\mathscr{B} \backslash\{U\}) \cup(N \backslash\{U\})$. Let $\lambda_{S}=\frac{\beta_{S}^{\mathcal{B}}}{1-\beta_{U}^{\mathcal{B}}}$ for all $S \in \mathscr{B} \backslash\{U\}, \lambda_{N \backslash U}=$ $-\frac{\beta_{U}^{\mathcal{B}}}{1-\beta_{U}^{\mathcal{B}}}$ and $\lambda_{S}=0$ for $S \in \mathcal{N} \backslash \mathcal{E}$. From Theorem 4.3, it follows that $\lambda \in \Lambda(\mathcal{E})$ and therefore $\varepsilon \in \mathbb{E}^{N}$.

We prove that $\mathscr{E} \in \mathbb{E}_{\min }^{N}$. Assume on the contrary that there exists a subset $\mathscr{D} \subsetneq \mathcal{E}$, with $\mathscr{D} \in \mathbb{E}_{\text {min }}^{N}$. By minimality of $\mathcal{B}$, it must hold that $N \backslash U \in \mathscr{D}$ as otherwise $\mathscr{D} \subsetneq \mathscr{B}$ which would be in contradiction with Theorem 4.1.

We distinguish two cases:

(i) Assume $\lambda_{S}^{\mathbb{D}}>0$ for all $S \in \mathscr{D} \backslash\{N \backslash U\}$. We know $\lambda_{N \backslash U}^{D}<1$, since $\lambda_{N \backslash U}^{D}=1$ would mean that $\mathscr{D} \backslash\{N \backslash U\}$ is a balanced collection on $U$ which contradicts minimality of $\mathcal{B}$ as we can omit $U$ from $\mathscr{B}$. Given that $\lambda_{N \backslash U}^{\mathcal{D}}<1$, we can reverse the procedure for constructing $\mathscr{E}$ : take $\mathscr{A}=(\mathscr{D} \backslash\{N \backslash U\}) \cup(\{U\})$ and take $\alpha_{S}=\frac{\lambda_{S}^{D}}{1-\lambda_{N \backslash U}^{D}}$ for all $S \in \mathscr{D} \backslash\{N \backslash U\}$ and $\alpha_{U}=-\frac{\lambda_{N \backslash U}^{D}}{1-\lambda_{N \backslash U}^{D}}$. We obtain $\alpha_{S}>0$ for all $S \in \mathcal{A} \backslash\{U\}$ and $\alpha_{U} \neq 0$. Furthermore, for $i \in U$ :

$$
\begin{aligned}
\sum_{S \in \mathcal{A}, S \ni i} \alpha_{S} & =\sum_{S \in \mathscr{D} \backslash\{N \backslash U\}, S \ni i} \frac{\lambda_{S}^{\mathcal{D}}}{1-\lambda_{N \backslash U}^{\mathbb{D}}}-\frac{\lambda_{N \backslash\{U\}}^{\mathcal{D}}}{1-\lambda_{N \backslash U}^{\mathcal{D}}} \\
& =\frac{1}{1-\lambda_{N \backslash U}^{\mathbb{D}}}-\frac{\lambda_{N \backslash U}^{\mathcal{D}}}{1-\lambda_{N \backslash U}^{\mathbb{D}}}=1,
\end{aligned}
$$

and for $i \in N \backslash U$ it holds that

$$
\sum_{S \in \mathcal{A}, S \ni i} \alpha_{S}=\sum_{S \in \mathcal{D} \backslash\{N \backslash U\}, S \ni i} \frac{\lambda_{S}^{\mathcal{D}}}{1-\lambda_{N \backslash U}^{\mathcal{D}}}=\frac{1-\lambda_{N \backslash U}^{\mathcal{D}}}{1-\lambda_{N \backslash U}^{\mathcal{D}}}=1 .
$$

So, $\alpha \in \Lambda(\mathcal{A})$ and therefore $\mathcal{A} \in \mathbb{E}^{N}$. As $\mathcal{A} \subsetneq \mathscr{B}$ this contradicts our assumption of $\mathscr{B} \in \mathbb{E}_{\text {min }}^{N}$.

(ii) Assume $\lambda_{T}^{\mathscr{D}}<0$ for some $T \in \mathscr{D} \backslash\{N \backslash U\}$, which means that $\mathscr{D} \in \mathbb{E}_{\min }^{N} \backslash \mathbb{B}_{\min }^{N}$ and $\lambda_{N \backslash U}^{\mathcal{D}}>0$. Take $c=-\frac{\lambda_{N \backslash U}}{\lambda_{N \backslash U}^{\mathcal{D}}}$, and take $T \in \mathscr{D}$ such that $\lambda_{T}^{\mathscr{D}}<0$. We construct the weight vector $\gamma$ with $\gamma_{S}=\frac{c}{1+c} \beta_{S}+\frac{1}{1+c} \lambda_{S}^{D}$ for all $S \in \mathcal{E}$ and $\gamma_{S}=0$ if $S \in \mathcal{N} \backslash \mathscr{E}$. Furthermore, take $\mathcal{C}=\left\{S \in \mathcal{E} \mid \gamma_{S} \neq 0\right\}$. By definition of $\beta$, we obtain $\gamma_{N \backslash U}=0$ and $\gamma_{S}>0$ for all $S \in \mathcal{C} \backslash\{T\}$. So, $\mathcal{C} \subsetneq \mathscr{B}$ and $\gamma \in \Lambda(\mathcal{C})$ so we obtain a contradiction with the minimality of $\mathcal{B}$.

So, we have $\mathscr{E} \in \mathbb{E}_{\mathrm{min}}^{N}$. From Theorem 3.7 it follows that $\Lambda(\mathscr{E})=$ $\{\lambda\}$. Since $\lambda_{N \backslash U}<0, \varepsilon \in \mathbb{E}_{\min }^{N} \backslash \mathbb{B}_{\min }^{N}$.

Proof of (ii). Let $\& \in \mathbb{E}_{\text {min }}^{N} \backslash \mathbb{B}_{\text {min }}^{N}$ and $N \notin \mathcal{E}$. Take $T \in \mathcal{E}$ such that $\lambda_{T}^{\mathscr{E}}<0$. Take $\mathcal{B}=(\mathscr{E} \backslash\{T\}) \cup\{N \backslash T\}$ and define $\beta_{S}=\frac{\lambda_{S}^{\varepsilon}}{1-\lambda_{T}^{\varepsilon}}$ for all $S \in \mathcal{E} \backslash\{T\}$ and $\beta_{N \backslash T}=-\frac{\lambda_{T}^{\varepsilon}}{1-\lambda_{T}^{\varepsilon}}$. We obtain $\beta_{S}>0$ for all $S \in \mathscr{B}$. Furthermore, for $i \in N \backslash T$ :

$\sum_{S \in \mathcal{B}, S \ni i} \beta_{S}=\sum_{S \in \mathcal{E}, S \ni i} \frac{\lambda_{S}^{\mathcal{E}}}{1-\lambda_{T}^{\mathcal{E}}}+\beta_{N \backslash T}=\frac{1}{1-\lambda_{T}^{\mathcal{E}}}-\frac{\lambda_{T}^{\mathcal{E}}}{1-\lambda_{T}^{\mathcal{E}}}=1$,

and for $i \in T$ it holds that

$\sum_{S \in \mathcal{B}, S \ni i} \beta_{S}=\sum_{S \in \mathcal{E} \backslash\{T\}, S \ni i} \frac{\lambda_{S}^{\mathcal{E}}}{1-\lambda_{T}^{\mathcal{E}}}=\frac{1-\lambda_{T}^{\mathcal{E}}}{1-\lambda_{T}^{\mathcal{E}}}=1$.

So, $\mathscr{B} \in \mathbb{B}^{N}$. It remains to show that $\mathscr{B}$ is minimal. Here we need the condition that $N \notin \mathcal{E}$, since there is no minimal balanced collection that contains $N$. If $\mathscr{B}$ is not minimal, then there exists a $\mathscr{B}^{\prime} \in \mathbb{B}_{\text {min }}^{N}$ such that $\mathscr{B}^{\prime} \subsetneq \mathscr{B}$. More precisely, as every balanced collection is the union of minimal balanced collections there exists a $\mathscr{B}^{\prime} \in \mathbb{B}_{\min }^{N}$ such that $N \backslash T \in \mathscr{B}^{\prime}$.
First suppose there exists a $\beta^{\prime} \in \Lambda^{+}\left(\mathscr{B}^{\prime}\right)$ such that $\beta_{N \backslash T}^{\prime}<1$. Then we obtain by definition of $\overline{\mathbb{B}}_{\text {min }}^{N}$ that $\left(\mathscr{B}^{\prime} \backslash\{N \backslash T\}\right) \cup(\{T\}) \in$ $\overline{\mathbb{B}}_{\text {min }}^{N} \subseteq \mathbb{E}_{\text {min }}^{N} \backslash \mathbb{B}_{\text {min }}^{N}$. Consequently, we have $\mathscr{B}^{\prime} \backslash\{N \backslash T\} \cup\{T\} \subsetneq \mathcal{E}$, a contraction with the minimality of $\mathcal{E}$.

Next suppose that for every minimal balanced collection $\mathcal{C} \subsetneq \mathscr{B}$ with $(N \backslash T) \in \mathcal{C}$ it holds that $\beta_{N \backslash T}^{\mathcal{C}}=1$. Take such a minimal balanced collection $\mathcal{C} \subsetneq \mathscr{B}$ with $(N \backslash T) \in \mathcal{C}$. We define a new collection $\mathcal{D}=\mathcal{C} \backslash\{N \backslash T\}$. Since $N \backslash T \notin \mathscr{D}$, we have $\mathscr{D} \subsetneq \mathcal{E}$. Also, $\sum_{S \in \mathcal{B}} \beta_{S}^{\mathcal{C}} e^{S}=e^{N}$ and therefore $\sum_{S \in \mathcal{D}} \beta_{S}^{\mathrm{C}} e^{S}=e^{N}-\beta_{N \backslash T}^{\mathrm{e}} e^{N \backslash T}=e^{T}$. This contradicts the minimality of $\mathscr{E}$, since we can take $\delta_{S}=\beta_{S}^{e}$ for every $S \in \mathscr{D}$ and $\delta_{T}=-1$, and we have $(1-\epsilon) \beta+\epsilon \delta \in \Lambda(\mathscr{E})$ for small $\epsilon>0$.

The third part of the partition consists of the minimal subbalanced collections. These collections consist of all minimal balanced collections of a subgame, to which the grand coalition of both the subgame and the original game are added.

For every $M \subsetneq N$ such that $|M| \geq 2$, define

$\widetilde{\mathbb{B}}_{\text {min }}^{N}(M)=\left\{\mathscr{B} \cup\{M, N\} \mid \mathscr{B} \in \mathbb{B}_{\min }^{M}\right\}$.

Also, define

$\widetilde{\mathbb{B}}_{\min }^{N}=\cup_{M \subsetneq N,|M| \geq 2} \widetilde{\mathbb{B}}_{\min }^{N}(M)$,

as the set of all minimal subbalanced collections.

For every minimal subbalanced collection, we can relate the weight vector of the underlying minimal balanced collection of a subgame to an exact balanced weight vector. For all coalitions in the balanced collection, the weight in the exact balanced weight vector is equal to the weight in the underlying balanced weight vector. The weight on the grand coalition of the subgame equals -1 in the exact balanced weight vector, and lastly the weight on the grand coalition in the original game equals 1 .

Theorem 4.5. Let $\varepsilon \in \widetilde{\mathbb{B}}_{\text {min }}^{N}$. Let $M \subsetneq N$ and $\mathscr{B} \in \mathbb{B}_{\text {min }}^{M}$ be such that $\mathcal{E}=(\mathscr{B} \cup\{M, N\})$. Let $\lambda_{S}=\beta_{S}^{\mathscr{B}}$ for all $S \in \mathscr{B}, \lambda_{M}=-1, \lambda_{N}=1$ and $\lambda_{S}=0$ for all $S \in \mathcal{N} \backslash \mathcal{E}$. Then $\lambda \in \Lambda(\mathcal{E})$.

Proof. It is readily checked that $\sum_{S \in \mathcal{E}} \lambda_{S} e^{S}=\sum_{S \in \mathcal{B}} \beta_{S}^{\mathcal{B}} e^{S}-e^{M}+$ $e^{N}=e^{N}$ and $\lambda_{S}>0$ for all $S \in \mathscr{E} \backslash\{M\}$. Hence, $\lambda \in \Lambda(\mathcal{E})$.

The following theorem shows that every minimal subbalanced collection is minimal exact balanced. Also, every minimal exact balanced collection that contains the grand coalition is a minimal subbalanced collection. This also means that the weight vector of Theorem 4.5 coincides with the standardized weight vector as introduced in Section 3.

\section{Theorem 4.6.}

(i) $\widetilde{\mathbb{B}}_{\min }^{N} \subseteq \mathbb{E}_{\min }^{N} \backslash \mathbb{B}_{\min }^{N}$,

(ii) Let $\varepsilon \in \mathbb{E}_{\min }^{N} \backslash \mathbb{B}_{\min }^{N}$ and $N \in \mathcal{E}$. Then $\varepsilon \in \widetilde{\mathbb{B}}_{\min }^{N}$.

Proof of (i). Let $\varepsilon \in \widetilde{\mathbb{B}}_{\text {min }}^{N}$. Let $M \subsetneq N$ and $\mathscr{B} \in \mathbb{B}_{\text {min }}^{M}$ be such that $\mathscr{E}=(\mathscr{B} \cup\{M, N\})$. Let $\lambda_{S}=\beta_{S}^{\mathcal{B}}$ for all $S \in \mathscr{B}, \lambda_{M}=-1, \lambda_{N}=1$ and $\lambda_{S}=0$ for all $S \in \mathcal{N} \backslash \mathscr{E}$. Theorem 4.5 shows that $\lambda \in \Lambda(\mathscr{E})$, so $\& \in \mathbb{E}^{N}$.

Suppose $\mathcal{E} \notin \mathbb{E}_{\text {min }}^{N}$. Take $\mathcal{D} \subsetneq \mathcal{E}$ such that $\mathscr{D} \in \mathbb{E}_{\text {min }}^{N}$. We have $N \in \mathscr{D}$ since the players in $N \backslash M$ are not present in any other coalition in $\mathcal{E}$. This also implies that $\lambda_{N}^{D}=1$. As $\{N\} \notin \mathbb{E}^{N}$ we have $\sum_{S \in \mathscr{D} \backslash\{N\}} \lambda_{S}^{\mathscr{D}} e^{S}=0$. This means that there exists a $T \in \mathscr{D} \backslash\{N\}$ such that $\lambda_{T}^{\mathcal{D}}<0$ and $S \subseteq T$ for all $S \in \mathscr{D} \backslash\{N\}$. Hence, $\sum_{S \in \mathscr{D} \backslash\{N, T\}}-\frac{\lambda_{S}}{\lambda_{T}} e^{S}=e^{T}$, so $\mathscr{D} \backslash\{N, T\} \in \mathbb{B}^{T}$.

First, suppose $T=M$. Then $\mathscr{D} \subsetneq \mathcal{E}$ gives $\mathscr{D} \backslash\{N, M\} \subsetneq \mathscr{B}$ which contradicts $\mathcal{B} \in \mathbb{B}_{\min }^{M}$.

Second, suppose $T \neq M$. Note that $S \subseteq M$ for every $S \in \mathscr{D} \backslash\{N\}$ as $\mathscr{D} \backslash\{M, N\} \subseteq \mathscr{B}$ and $\mathcal{B} \in \mathbb{B}_{\min }^{M}$. So in particular $T \subsetneq M$. On the other hand, we have shown that $S \subseteq T$ for all $S \in \mathscr{D} \backslash\{N\}$. 
Hence, $M \notin \mathscr{D}$ and $\mathscr{D} \backslash\{N\} \subset \mathscr{B}$. Define the weight vector $\delta$ such that $\delta_{S}=\lambda_{S}^{D}$ for all $S \in \mathscr{D}^{\mp} \backslash\{N\}$ and $\delta_{S}=0$ otherwise. Now, for small $\epsilon>0$ we have $\epsilon \lambda^{\mathscr{D}}+\beta^{\mathscr{B}} \in \Lambda^{+}(\mathscr{B})$ which contradicts Theorem 2.7.

Proof of (ii). By Theorem 3.9 we have $\lambda_{N}^{\mathcal{E}}=1$. Take $T \in \mathcal{E}$ such that $\lambda_{T}^{\mathcal{E}}=-1$. We have $\sum_{S \in \mathcal{E} \backslash\{N\}} \lambda_{S} e^{S}=0$ which yields $\sum_{S \in \mathscr{E} \backslash\{N, T\}} \lambda_{S} e^{S}=e^{T}$, and therefore $\mathcal{E} \backslash\{N, T\} \in \mathbb{B}^{T}$. If there exists a minimal balanced collection $\mathscr{B} \in \mathbb{B}_{\min }^{T}$ such that $\mathscr{B} \subsetneq \mathcal{E} \backslash\{T, N\}$, it is readily checked that $B \cup\{T, N\}$ is an exact balanced collection, which contradicts our assumption of $\mathcal{E} \in \mathbb{E}_{\min }^{N}$. Hence, $\mathcal{E} \backslash\{N, T\} \in$ $\mathbb{B}_{\min }^{T}$ and $\varepsilon \in \widetilde{\mathbb{B}}_{\min }^{N}$

The following corollary follows from Theorems 4.1, 4.4 and 4.6.

Corollary 4.7. The three sets $\widetilde{\mathbb{B}}_{\min }^{N}, \overline{\mathbb{B}}_{\min }^{N}$, and $\mathbb{B}_{\min }^{N}$ form a partition of $\mathbb{E}_{\mathrm{min}}^{N}$.

\section{Sufficient conditions for exactness}

As mentioned before, the class of minimal balanced collections is useful as one does not need other balanced collections to check whether a game is balanced. The class of minimal exact balanced weights exhibits the same feature: the following theorem shows that we only need the minimal exact balanced collections to check whether a game is exact.

Theorem 5.1. Let $v \in V(\varepsilon)$ for all $\& \in \mathbb{E}_{\min }^{N}$. Then $v \in V$.

Proof. Let $\mathscr{D} \in \mathbb{E}^{N} \backslash \mathbb{E}_{\min }^{N}$. Let $\delta \in \Lambda(\mathscr{D})$. It suffices to show that $v \in V(\delta)$ i.e., $\sum_{S \in \mathbb{D}} \delta_{S} v(S) \leq v(N)$.

First, assume $\delta \in \Lambda^{+}(\mathscr{D})$. Then Theorems 2.6 and 4.1 imply that $v \in V(\delta)$.

Second, assume that $\delta \notin \Lambda^{+}(\mathscr{D})$. Take $U \in \mathscr{D}$ such that $\delta_{U}<0$.

If $U=N$, then define $\mathcal{C}=\mathscr{D} \backslash\{N\}$ and $\gamma_{S}=\frac{\delta_{S}}{1-\delta_{N}}$ for all $S \in \mathcal{C}$ and $\gamma_{S}=0$ for all $S \in \mathcal{N} \backslash \mathcal{C}$. We have $\gamma \in \Lambda^{+}(\mathcal{C})$ and $\mathcal{C} \in \mathbb{B}^{N}$. Note that $v \in V(\delta)$ is directly implied by $v \in V(\gamma)$. Hence, in the remainder we will assume that $U \neq N$.

Since $\mathscr{D} \notin \mathbb{E}_{\text {min }}^{N}$, we can take $\mathcal{A} \in \mathbb{E}_{\min }^{N}$ such that $\mathcal{A} \subsetneq \mathcal{D}$.

Case 1: $\lambda_{U}^{\mathcal{A}} \geq 0$, so either $\lambda_{U}^{\mathcal{A}}>0$ or $U \notin \mathcal{A}$. If $\mathcal{A} \in \mathbb{E}_{\text {min }}^{N} \backslash \mathbb{B}_{\text {min }}^{N}$, then take $T \in \mathcal{A}$ such that $\lambda_{T}^{\mathcal{A}}<0$. If $\mathcal{A} \in \mathbb{B}_{\text {min }}^{N}$, define $T=\emptyset$. Define $a=\min \left\{\frac{\lambda_{S}}{\lambda_{S}^{4}} \mid S \in \mathcal{A} \backslash\{T, U\}\right\}$. We first show that $a \leq 1$.

Suppose on the contrary that $a>1$. As $\delta_{S}>\lambda_{S}^{\mathcal{A}}$ for every $S \in \mathscr{D} \backslash\{U\}$, we have for $i \in N \backslash U$ that

$\sum_{\substack{S \in \mathcal{D}, S \ni i}} \delta_{S}=\sum_{\substack{S \in \mathcal{D} \backslash\{U\}, S \ni i}} \delta_{S}>\sum_{\substack{S \in \mathcal{D} \backslash\{U\}, S \ni i}} \lambda_{S}^{\mathcal{A}}=1$,

a contradiction.

If we can find an $\mathcal{A} \in \mathbb{E}^{N}$ such that $\mathcal{A} \subsetneq \mathcal{D}$ and $\cap_{\mathcal{E} \in \mathbb{E}_{\min }^{N}} V(\mathcal{E}) \cap$ $V(\mathcal{A}) \subseteq V(\delta)$, we may conclude that $\cap_{\mathcal{E} \in \mathbb{E}_{\min }^{N}} V(\mathscr{E}) \subseteq V(\delta)$. We discriminate between two subcases:

- $a=1$. If $T \backslash U \neq \emptyset$, then for $i \in T \backslash U$ it holds that

$\sum_{\substack{S \in \mathcal{D}, S \ni i}} \delta_{S}=\sum_{\substack{S \in \mathcal{D} \backslash\{T, U\}, S \ni i}} \lambda_{S}+\delta_{T}>\sum_{\substack{S \in \mathcal{D} \backslash\{T, U\}, S \ni i}} \lambda_{S}^{\mathcal{A}}+\lambda_{T}^{\mathcal{A}}=1$,

which cannot hold. On the other hand, if $T \backslash U=\emptyset$, define $\kappa_{S}=\frac{\delta_{S}-\lambda_{S}^{A}}{\delta_{U}^{A}-\delta_{U}}$ for all $S \in \mathscr{D}, \kappa_{N}=1$ and $\kappa_{S}=0$ for all $S \in$ $\mathcal{N} \backslash(\mathscr{D} \cup\{N\})$. Take $\mathcal{K}=\left\{S \in \mathscr{D} \cup\{N\} \mid \kappa_{S} \neq 0\right\}$. Now $V(\kappa) \cap V\left(\lambda^{\mathcal{A}}\right) \subseteq V(\delta)$ as from

$$
\begin{aligned}
\left(\lambda_{U}^{\mathcal{A}}-\delta_{U}\right) \sum_{S \in \mathcal{K}} \kappa_{S} v(S)= & \sum_{S \in \mathcal{D} \backslash\{U\}}\left(\delta_{S}-\lambda_{S}^{\mathcal{A}}\right) v(S) \\
& -\left(\lambda_{U}^{\mathcal{A}}-\delta_{U}\right) v(U)+\left(\lambda_{U}^{\mathcal{A}}-\delta_{U}\right) v(N) \\
\leq & \left(\lambda_{U}^{\mathcal{A}}-\delta_{U}\right) v(N),
\end{aligned}
$$

and $\sum_{S \in \mathcal{A}} \lambda_{S}^{\mathcal{A}} v(S) \leq v(N)$ it follows that $\sum_{S \in \mathscr{D}} \delta_{S} v(S) \leq v(N)$.
Note that $\mathcal{K} \backslash\{N, U\} \in \mathbb{B}^{U}$ as $\kappa_{S}>0$ for all $S \in \mathcal{K} \backslash\{N, U\}$ and

$$
\begin{aligned}
\sum_{S \in \mathcal{K}} \kappa_{S} e^{S} & =\sum_{S \in \mathcal{D} \backslash\{U\}} \delta_{S} e^{S}-\sum_{S \in \mathcal{D} \backslash\{U\}} \lambda_{S}^{\mathcal{A}} e^{S} \\
& =\frac{1}{\lambda_{U}^{\mathcal{A}}-\delta_{U}}\left(\left(e^{N}-\delta_{U} e^{U}\right)-\left(e^{N}-\lambda_{U}^{\mathcal{A}} e^{U}\right)\right) \\
& =e^{U} .
\end{aligned}
$$

- $a<1$. We define $\kappa_{S}=\frac{1}{1-a} \delta_{S}-\frac{a}{1-a} \lambda_{S}^{\mathcal{A}}$ for all $S \in \mathscr{D}, \kappa_{S}=0$ for $S \in \mathcal{N} \backslash \mathscr{D}$ and define $\mathcal{K} \stackrel{1}{=}\left\{S \in \mathscr{D} \mid \kappa_{S} \neq 0\right\}$. By definition of $a$, we obtain $\mathcal{K} \subsetneq \mathcal{D}$. It is now easily seen that $V(\kappa) \cap V\left(\lambda^{\mathcal{A}}\right) \subseteq V(\delta)$, as

$\sum_{S \in \mathcal{D}} \delta_{S} v(S)=(1-a) \sum_{S \in \mathcal{K}} \kappa_{S} v(S)+a \sum_{S \in \mathcal{A}} \lambda_{S}^{\mathcal{A}} v(S) \leq v(N)$.

Note that $\mathcal{K} \in \mathbb{E}^{N}$ and $\kappa \in \Lambda(\mathcal{K})$, as $\kappa_{S}>0$ for all $S \in$ $\mathcal{K} \backslash\{U\}, \kappa_{U}<0$, and

$$
\begin{aligned}
\sum_{S \in \mathcal{K}} \kappa_{S} e^{S} & =\sum_{S \in \mathcal{D}}\left(\frac{1}{1-a} \delta_{S}-\frac{a}{1-a} \lambda_{S}^{\mathcal{A}}\right) e^{S} \\
& =\frac{1}{1-a} \sum_{S \in \mathscr{D}} \delta_{S} e^{S}-\frac{a}{1-a} \sum_{S \in \mathcal{A}} \lambda_{S}^{\mathcal{A}} e^{S}=e^{N} .
\end{aligned}
$$

Case 2: $\lambda_{U}^{\mathcal{A}}<0$. Take $a=\min \left\{\frac{\delta_{S}}{\lambda_{S}^{\mathcal{A}}} \mid S \in \mathcal{A}\right\}$. It holds that $a<1$, as $a=1$ would imply that $\delta_{S}=\lambda_{S}^{\mathcal{A}}$ for all $S \in \mathcal{A}$ which implies $\delta_{S}=0$ for all $S \in \mathscr{D} \backslash \mathcal{A}$. Furthermore, $a>1$ would imply that $\sum_{S \in \mathcal{D}, S \ni i} \delta_{S}>\sum_{S \in \mathcal{A}, S \ni i} \lambda_{S}^{\mathcal{A}}=1$ for $i \in N \backslash U$.

Again, if we can find an $\mathcal{A} \in \mathbb{E}^{N}$ such that $\mathcal{A} \subsetneq \mathscr{D}$ and $\cap_{\mathscr{E} \in \mathbb{E}_{\min }^{N}} V(\mathscr{E}) \cap V(\mathcal{A}) \subseteq V(\delta)$, we may conclude that $\cap_{\mathscr{E} \in \mathbb{E}_{\min }^{N}} V(\mathscr{E}) \subseteq V(\delta)$. We construct $\kappa_{S}=\frac{1}{1-a} \delta_{S}-\frac{a}{1-a} \lambda_{S}^{\mathcal{A}}$ for all $S \in \mathscr{D}$ and $\mathcal{K}=\left\{S \in \mathscr{D} \mid \kappa_{S} \neq 0\right\}$. We have $V(\kappa) \cap V\left(\lambda^{\mathcal{A}}\right) \subseteq V(\delta)$, as

$\sum_{S \in \mathcal{D}} \delta_{S} v(S)=(1-a) \sum_{S \in \mathcal{K}} \kappa_{S} v(S)+a \sum_{S \in \mathcal{A}} \lambda_{S}^{\mathcal{A}} v(S) \leq v(N)$

We have $\mathcal{K} \in \mathbb{E}^{N}$ and $\kappa \in \Lambda(\mathcal{K})$ since $\kappa_{S} \geq 0$ for all $S \in \mathcal{K} \backslash\{U\}$ and $\sum_{S \in \mathcal{K}} \kappa_{S} e^{S}=e^{N}$.

The equivalent of Theorem 2.8 for minimal exact balanced collections however does not hold, as there exist minimal exact balanced collections that are redundant. The following example illustrates this.

Example 5.2. Consider the minimal exact balanced collections $\mathscr{B}=\{\{1,2\},\{1,3\},\{2,3\}\}$ with weight vector $\beta^{\mathcal{B}}$ such that $\beta_{\{1,2\}}^{\mathscr{B}}=\beta_{\{1,3\}}^{\mathscr{B}}=\beta_{\{2,3\}}^{\mathcal{B}}=\frac{1}{2}, \mathcal{C}=\{\{1\},\{2,3\}\}$ with weights $\beta_{\{1\}}^{\mathrm{e}}=\beta_{\{2,3\}}^{\mathrm{e}}=1$ and $\mathcal{E}=\{\{1,2\},\{1,3\},\{1\}\}$ with $\lambda_{\{1,2\}}^{\mathcal{E}}=$ $\lambda_{\{1,3\}}^{\mathcal{E}}=1$ and $\lambda_{\{1\}}^{\mathcal{E}}=-1$. We have $V(\mathcal{C}) \cap V(\mathcal{E}) \subseteq V(\mathscr{B})$, since $\beta^{\mathscr{B}}=\frac{1}{2} \beta^{\mathcal{C}}+\frac{1}{2} \lambda^{\mathcal{E}}$.

The question arises which minimal exact balanced collections

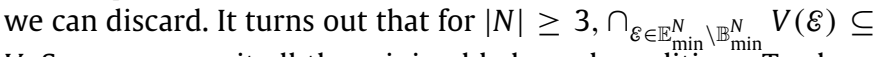
$V$. So, we can omit all the minimal balanced conditions. To show this, we first introduce a lemma to construct particular members of $\mathbb{E}_{\min }^{N}$.

Lemma 5.3. Let $|N| \geq 3$ and take $S \in \mathcal{N}$ and $T \in \mathcal{N}$ such that $S \cap T=\emptyset$.

(i) If $S \cup T=N,|T| \geq 2$ and $i \in T$, then $\{S \cup\{i\}, T,\{i\}\} \in$ $\mathbb{E}_{\text {min }}^{N} \backslash \mathbb{B}_{\text {min }}^{N}$.

(ii) If $S \cup T \neq N$, then $\{S, T, S \cup T, N\} \in \mathbb{E}_{\min }^{N} \backslash \mathbb{B}_{\min }^{N}$. 
Proof of (i). The collection $\{S \cup\{i\}, T, N \backslash\{i\}\}$ is minimal balanced with weight vector $\lambda$ such that $\lambda_{S \cup\{i\}}=\lambda_{T}=\lambda_{N \backslash\{i\}}=\frac{1}{2}$. By definition of $\overline{\mathbb{B}}_{\min }^{N}$, we have $\{S \cup\{i\}, T,\{i\}\} \in \overline{\mathbb{B}}_{\min }^{N}$. By Theorem 4.4 this means that $\{S \cup\{i\}, T,\{i\}\} \in \mathbb{E}_{\text {min }}^{N} \backslash \mathbb{B}_{\text {min }}^{N}$.

Proof of (ii). The collection $\{S, T\}$ is minimal balanced for player set $S \cup T$. By definition of $\widetilde{\mathbb{B}}_{\min }^{N}(S \cup T)$, we have $\{S, T, S \cup T, N\} \in$ $\widetilde{\mathbb{B}}_{\min }^{N}(S \cup T)$. By Theorem 4.6 this means that $\{S, T, S \cup T, N\} \in$ $\mathbb{E}_{\min }^{N} \backslash \mathbb{B}_{\min }^{N}$.

Theorem 5.4. Let $v \in V(\mathscr{E})$ for every $\& \in \mathbb{E}_{\min }^{N} \backslash \mathbb{B}_{\min }^{N}$ and $|N| \geq 3$. Then $v \in V$.

Proof. Let $\mathscr{B} \in \mathbb{B}_{\min }^{N}$. First, consider the case where $\mathscr{B}$ is a partition. Assume $\mathscr{B}=\{S, T\}$ for some $S, T \in 2^{N} \backslash\{\emptyset\}$. Note that $\beta_{S}^{\mathscr{B}}=\beta_{T}^{\mathcal{B}}=1$. Without loss of generality, we assume $|S| \leq|T|$. Take $i \in T, \mathcal{A}=\{\{i\}, S, S \cup\{i\}, N\}$ with $\lambda_{\{i\}}^{\mathcal{A}}=\lambda_{S}^{\mathcal{A}}=\lambda_{N}^{\mathcal{A}}=1$ and $\lambda_{S \cup\{i\}}^{\mathcal{A}}=-1$ and $\mathscr{D}=\{S \cup\{i\}, T,\{i\}\}$ with $\lambda_{S \cup\{i\}}^{\mathcal{D}}=\lambda_{T}^{D}=1$ and $\lambda_{\{i\}}^{D}=-1$. By Lemma 5.3, $\mathcal{A} \in \mathbb{E}_{\text {min }}^{N} \backslash \mathbb{B}_{\text {min }}^{N}$ and $\mathscr{D} \in \mathbb{E}_{\text {min }}^{N} \backslash \mathbb{B}_{\text {min }}^{N}$. Now $v \in V^{+}(\mathscr{B})$ follows from $v \in V(\mathscr{A})$ and $v \in V(\mathscr{D})$ : from

$v(\{i\})+v(S)-v(S \cup\{i\})+v(N) \leq v(N)$,

and

$v(S \cup\{i\})+v(T)-v(\{i\}) \leq v(N)$,

it follows that

$v(S)+v(T) \leq v(N)$.

We show that for every partition $\mathscr{B}$ with $|\mathscr{B}| \geq 3$ there exists a

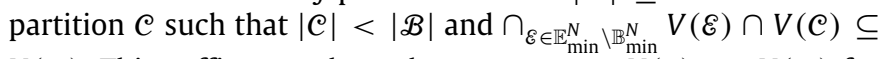

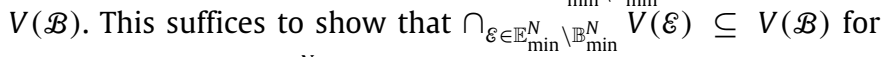
every partition $\mathscr{B} \in \mathbb{B}_{\text {min }}^{N}$.

Assume that $\mathscr{B}$ is a partition of the player set $N$, with $|\mathscr{B}| \geq 3$. Take $S \in \mathscr{B}$ and $T \in \mathscr{B}$ with $S \neq T$. Define $\mathcal{A}=\{S, T, S \cup T, N\}$ with $\lambda_{S}^{\mathcal{A}}=\lambda_{T}^{\mathcal{A}}=\lambda_{N}^{\mathcal{A}}=1$ and $\lambda_{S \cup T}^{\mathcal{A}}=-1$. By Lemma 5.3 we have $\mathscr{A} \in \mathbb{E}_{\text {min }}^{N} \backslash \mathbb{B}_{\text {min }}^{N}$. Define $\mathscr{D}=(\mathscr{B} \backslash\{S, T\}) \cup\{S \cup T\}$ and $\delta \in \Lambda(\mathscr{D})$ such that $\delta_{S}=1$ for all $S \in \mathscr{D}$. It is readily checked that $V(\mathscr{D}) \cap V(\mathscr{A}) \subseteq V(\mathscr{B})$. Furthermore, $\mathscr{D}$ is a partition and $|\mathcal{D}|<|\mathcal{B}|$.

Second, consider the case where $\mathscr{B}$ is not a partition. Take $T \in$ $\mathscr{B}$ such that $\beta_{T}^{\mathscr{B}}<1$. As $\mathscr{B}$ is not a partition, such a coalition exists and $N \backslash T \notin \mathscr{B}$. Define $\mathcal{C}=\{T, N \backslash T\}$ and $\mathscr{D}=(\mathscr{B} \backslash\{T\}) \cup\{N \backslash T\}$ with $\delta_{S}=\frac{\beta_{S}^{\mathscr{B}}}{1-\beta_{T}^{\mathscr{B}}}$ for all $S \in \mathcal{B} \backslash\{T\}$ and $\delta_{N \backslash T}=-\frac{\beta_{T}^{\mathcal{B}}}{1-\beta_{T}^{\mathcal{B}}}$. We have

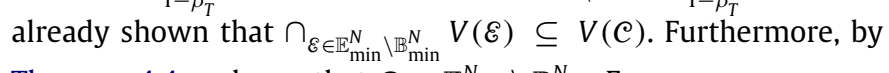
Theorem 4.4 we know that $\mathscr{D} \in \mathbb{E}_{\text {min }}^{N} \backslash \mathbb{B}_{\text {min }}^{N}$. From

$\beta_{T}^{\mathcal{B}}[v(T)+v(N \backslash T)] \leq \beta_{T}^{\mathcal{B}} v(N)$,

and

$$
\begin{aligned}
& \left(1-\beta_{T}^{\mathcal{B}}\right)\left(\sum_{S \in \mathcal{D} \backslash(N \backslash T)} \frac{\beta_{S}^{\mathcal{B}}}{1-\beta_{T}^{\mathcal{B}}} v(S)-\frac{\beta_{T}^{\mathcal{B}}}{1-\beta_{T}^{\mathcal{B}}} v(N \backslash T)\right) \\
& \leq\left(1-\beta_{T}^{\mathcal{B}}\right) v(N),
\end{aligned}
$$

it follows that

$\sum_{S \in \mathscr{B}} \beta_{S}^{\mathcal{B}} v(S) \leq v(N)$.

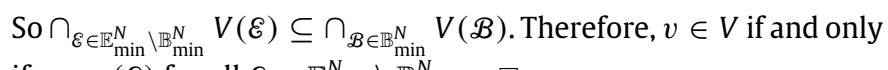
if $v \in v(\mathcal{E})$ for all $\& \in \mathbb{E}_{\min }^{N} \backslash \mathbb{B}_{\min }^{N}$.

We have shown that for verifying that a game is exact, the class of minimal balanced collections is redundant. However, as the following example demonstrates, there exists an even smaller subclass of the class of minimal exact balanced collections that still ensures exactness of the game.
Example 5.5. Let $N=\{1,2,3,4\}$. Consider the minimal exact balanced collections $\mathcal{A}=\{\{2\},\{1,4\},\{1,2,4\}, N\}, \mathcal{D}=\{\{1,2,4\}$, $\{1,2,3\},\{1,2\}\}$ and $\mathscr{E}=\{\{2\},\{1,2\},\{1,4\},\{1,2,3\}\}$. From

$v(\{2\})+v(\{1,4\})-v(\{1,2,4\})+v(N) \leq v(N)$,

and

$v(\{1,2,4\})+v(\{1,2,3\})-v(\{1,2\}) \leq v(N)$,

we have that

$v(\{2\})+v(\{1,4\})+v(\{1,2,3\})-v(\{1,2\}) \leq v(N)$.

This implies that $V(\mathcal{A}) \cap V(\mathscr{D}) \subseteq V(\mathscr{E})$, so $\&$ is redundant.

Further research on the topic could possibly establish a characterization of a subclass of minimal exact balanced collections that is sharp, in the sense that no collection can be left out while still guaranteeing exactness.

\section{On the construction of minimal exact balanced collections}

Using Theorem 2.6, it can be checked if a game is balanced utilizing minimal balanced collections only. However, the efficiency of this approach depends on the construction of these collections. Peleg (1965) provides an efficient and comprehensive algorithm for obtaining all minimal balanced collections. Given a player set and the corresponding class of minimal balanced collections, the algorithm constructs from every minimal balanced collection a number of candidate collections for a player set with one player extra. By checking a number of basic conditions on the candidate collection and the weight vector of the collection on the smaller player set, it is readily checked if the candidate is indeed minimal.

This procedure can be extended to efficiently check for exactness of a game. As we derived an explicit relation between minimal balanced collections on the one hand and minimal negative balanced collections and minimal subbalanced collections on the other hand, the collections and their respective weight vectors can be constructed from the minimal balanced collections. Theorems 4.4 and 4.6 prove the relation between minimal balanced collections on the one hand and minimal negative balanced collections and minimal subbalanced collections on the other hand. Theorems 4.3 and 4.5 show how the exact balanced weight vectors can be obtained from minimal balanced weight vectors. Note that the minimal balanced collections of every subset of the player set, which are needed to construct the minimal subbalanced collections, are constructed by the Peleg procedure in the process.

\section{Acknowledgments}

The third author would like to thank the Netherlands Organisation for Scientific Research (NWO) for the financial support.

\section{References}

Bondareva, O., 1963. Some applications of linear programming methods to the theory of cooperative games. Problemy Kybernetiki 10, 119-139 (in Russian).

Branzei, R., Tijs, S., Zarzuelo, J., 2009. Convex multi-choice cooperative games: characterizations and monotonic allocation schemes. European Journal of Operational Research 198, 571-575.

Calleja, P., Borm, P., Hendrickx, R., 2005. Multi-issue allocation situations. European Journal of Operational Research 164, 730-747.

Csóka, P., Herings, P.J.J., Kóczy, L.Á., 2009. Stable allocations of risk. Games and Economic Behavior 67, 266-276.

Csóka, P., Herings, P.J.J., Kóczy, L.Á., 2011. Balancedness conditions for exact games. Mathematical Methods of Operations Research 74, 41-52.

Derks, J., Haller, H., 1999. Weighted nucleoli. International Journal of Game Theory $28,173-187$. 
Gillies, D., 1953. Some Theorems on $n$-Person Games. Princeton University Press, Princeton, New Jersey.

Kohlberg, E., 1971. On the nucleolus of a characteristic function game. SIAM Journal on Applied Mathematics 20, 62-66.

Peleg, B., 1965. An inductive method for constructing minimal balanced collections of finite sets. Naval Research Logistics Quarterly 12, 155-162.

Peleg, B., Sudhölter, P., 2003. Introduction to the Theory of Cooperative Games. Kluwer Academic Publishers, Dordrecht.
Schmeidler, D., 1969. The nucleolus of a characteristic function game. SIAM Journal on Applied Mathematics 17, 1163-1170.

Schmeidler, D., 1972. Cores of exact games. Journal of Mathematical Analysis and Applications 40, 214-225.

Shapley, L., 1967. On balanced sets and cores. Naval Research Logistics Quarterly 14 453-460.

Shapley, L., 1971. Cores of convex games. International Journal of Game Theory 1, 11-26. 